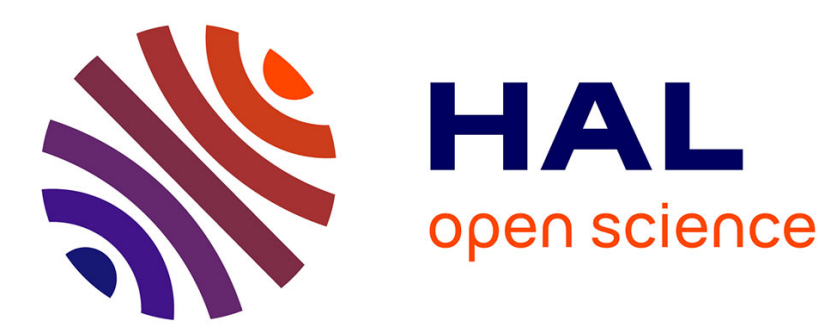

\title{
11. Mécanismes de pertes d'énergie des quasi-particules excitées dans un supraconducteur
}

\author{
C.J. Adkins, A.R. Long
}

\section{To cite this version:}

C.J. Adkins, A.R. Long. 11. Mécanismes de pertes d'énergie des quasi-particules excitées dans un supraconducteur. Revue de Physique Appliquée, 1970, 5 (6), pp.903-904. 10.1051/rphysap:0197000506090302 . jpa-00243481

HAL Id: jpa-00243481

https://hal.science/jpa-00243481

Submitted on 1 Jan 1970

HAL is a multi-disciplinary open access archive for the deposit and dissemination of scientific research documents, whether they are published or not. The documents may come from teaching and research institutions in France or abroad, or from public or private research centers.
L'archive ouverte pluridisciplinaire HAL, est destinée au dépôt et à la diffusion de documents scientifiques de niveau recherche, publiés ou non, émanant des établissements d'enseignement et de recherche français ou étrangers, des laboratoires publics ou privés. 


\title{
11. MÉCANISMES DE PERTES D'ÉNERGIE DES QUASI-PARTICULES EXCITÉES DANS UN SUPRACONDUCTEUR
}

\author{
C. J. ADKINS et A. R. LONG
}

Royal Society Mond Laboratory, Free School Lane, Cambridge, England

Quand une tension plus grande que $2 \Delta / e$ est appliquée à une jonction tunnel dont les électrodes sont des supraconducteurs avec une bande d'énergie interdite $\Delta$, des phonons d'énergie $2 \Delta$ peuvent être crées. Ceux-ci peuvent être détectés par une deuxième jonction tunnel. Le signal dans la jonction détectrice est proportionnel au courant dans la jonction émettrice jusqu'à ce que le potentiel de l'émetteur atteigne $4 \Delta$. La dérivée de la caractéristique de transfert présente une discontinuité augmentant de 2,5 fois la valeur initiale [1, 2]. L'accroissement du signal du détecteur peut être attribué à l'énergie perdue par l'émission de phonons par des quasi-particules dans l'émetteur. Si ce processus était le seul moyen de pertes d'énergie, un calcul détaillé montrerait que la variation de la dérivée serait de 3,25. Ceci implique que d'autres mécanismes de pertes d'énergie doivent intervenir. L'effet tunnel inélastique ne fournit pas une explication satisfaisante parce que le nombre de canaux permettant un processus inélastique qui serait exigé, est si important que la caractéristique $I-V$ du générateur serait fortement non linéaire ce qui n'est pas observé. Cependant, les collisions inélastiques des quasi-particules excitées avec la barrière doit fournir une explication possible.

En outre, il existe une difficulté dans le cas de l'aluminium lorsque le détecteur et le générateur possèdent une électrode commune. Pour une telle géométrie on devrait s'attendre à une diminution importante du rapport du gradient de la caractéristique de transfert alors qu'expérimentalement une légère réduction est obtenue. On peut expliquer ce point en admettant 
que l'énergie des quasi-particules excitées est partiellement dissipée dans le film. Un calcul du taux de pertes d'énergie par un processus direct de destruction des paires utilisant les données expérimentales des sections efficaces des collisions électron-électron [3] montre que la quantité d'énergie perdue par un processus direct de dépariement est probablement comparable à celle perdue par émission de phonons dans le cas de l'aluminium. Pour des supraconducteurs à cou- plage fort l'énergie perdue par destruction des paires est moins importante.

\section{Bibliographie}

[1] Eisenmenger (W.), Dayem (A. M.), Phys. Rev. Lettres, $1967,18-125$.

[2] Gray (K. E.), Thèse, Université de Cambridge, 1969.

[3] Garland (J. C.), Bowers (R.), Phys. Rev. Lettres. 1968, 21, 1007. 